\title{
Quest for absolute zero in the presence of external noise
}

\author{
E. Torrontegui ${ }^{1,2}$ and R. Kosloff ${ }^{2}$ \\ ${ }^{1}$ Departamento de Química-Física, Universidad del País Vasco-Euskal Herriko Unibertsitatea, Apdo. 644, Bilbao, Spain \\ ${ }^{2}$ Institute of Chemistry, The Hebrew University, Jerusalem 91904, Israel
}

(Received 22 May 2013; published 3 September 2013)

\begin{abstract}
A reciprocating quantum refrigerator is analyzed with the intention to study the limitations imposed by external noise. In particular we focus on the behavior of the refrigerator when it approaches the absolute zero. The cooling cycle is based on the Otto cycle with a working medium constituted by an ensemble of noninteracting harmonic oscillators. The compression and expansion segments are generated by changing an external parameter in the Hamiltonian. In this case the force constant of the harmonic oscillators $m \omega^{2}$ is modified from an initial to a final value. As a result, the kinetic and potential energy of the system do not commute causing frictional losses. By proper choice of scheduling function $\omega(t)$ frictionless solutions can be obtained in the noiseless case. We examine the performance of a refrigerator subject to noise. By expanding from the adiabatic limit we find that the external noise, Gaussian phase, and amplitude noises reduce the amount of heat that can be extracted but nevertheless the zero temperature can be approached.
\end{abstract}

DOI: 10.1103/PhysRevE.88.032103

PACS number(s): 05.30.-d, 05.70.Ln, 07.20.Pe

\section{THE SYSTEM}

Reciprocating refrigerators operate by a working medium shuttling heat from the cold bath at $T_{c}$ temperature to the hot reservoir with $T_{h}$ temperature. The task is carried out by a controlled dynamical system. By changing the Hamiltonian of the system the internal levels of the working medium can be modified. Upon contact with the cold side the internal energy of the working medium is forced to be lower than the equilibrium energy associated with the $T_{c}$ temperature, only under these conditions heat flows from the cold to the heat bath [1]. In the hot reservoir, however, a reciprocal relation is required. In the present work we consider a refrigerator using a controllable quantum medium as its working medium [2-7]. The medium is constituted by an ensemble of noninteracting particles bound by a shared harmonic potential whose frequency $\omega(t)$ can be controlled and varies between two extreme values $\omega_{c}$ and $\omega_{h}$, the frequencies associated with the cold and hot baths, respectively [2-4]. The energy of each particle is represented by the Hamiltonian

$$
\hat{\mathbf{H}}=\frac{1}{2 m} \hat{\mathbf{P}}^{2}+\frac{1}{2} m \omega(t)^{2} \hat{\mathbf{Q}}^{2},
$$

where $m$ is the mass of each particle, and $\hat{\mathbf{P}}$ and $\hat{\mathbf{Q}}$ are the momentum and position operators.

The dynamics of the quantum thermodynamical observables are described within the formalism of quantum open systems generated by the Liouville superoperator $\mathcal{L}$ studied in the Heisenberg picture $[8,9]$,

$$
\frac{d \hat{\mathbf{A}}}{d t}=\frac{i}{\hbar}[\hat{\mathbf{H}}, \hat{\mathbf{A}}]+\mathcal{L}(\hat{\mathbf{A}})+\frac{\partial \hat{\mathbf{A}}}{\partial t},
$$

where $\mathcal{L}$ is a generator of a completely positive Liouville superoperator and $[\hat{A}, \hat{B}]=\hat{A} \hat{B}-\hat{B} \hat{A}$. Typically, $\left[\hat{\mathbf{H}}(t), \hat{\mathbf{H}}\left(t^{\prime}\right)\right] \neq 0$ which leads to frictionlike phenomena $[2,7]$, too fast adiabatic segments will generate parasitic internal energy which will have to be dissipated to the heat baths limiting the performance.

The considered cycle is composed [2,3] by two segments, termed isochores, where the working medium is in contact with the cold or hot baths and the external control field $\omega$ is maintained constant. There are also two other segments, termed adiabats, where the working medium is isolated from the baths and the frequency $\omega(t)$ is modified. This represents the quantum version of the Otto cycle. Each segment is characterized by a quantum propagator $\mathcal{U}_{s}$ which maps the initial state of the working medium to the final one. At the isochores $\mathcal{L}=\mathcal{L}_{D}$ is the dissipative term responsible for driving the working medium to thermal equilibrium when it is in contact with either the cold or hot baths. For the harmonic oscillator $\mathcal{L}_{D}$ takes the explicit form [2]

$$
\mathcal{L}_{D}(\hat{\rho})=k_{\downarrow}\left(\hat{a}^{\dagger} \hat{\rho} \hat{a}-\left\{\hat{a} \hat{a}^{\dagger}, \hat{\rho}\right\} / 2\right)+k_{\uparrow}\left(\hat{a} \hat{\rho} \hat{a}^{\dagger}-\left\{\hat{a}^{\dagger} \hat{a}, \hat{\rho}\right\} / 2\right),
$$

where $\{\hat{A}, \hat{B}\}=\hat{A} \hat{B}+\hat{B} \hat{A}, \hat{a}$ and $\hat{a}^{\dagger}$ are the lowering and raising operators of the harmonic oscillator, and $k_{\downarrow}$ and $k_{\uparrow}$ are the heat conductances satisfying detailed balance $k_{\uparrow} / k_{\downarrow}=$ $\exp \left[-\hbar \omega /\left(k_{B} T\right)\right]$ with $T$ either $T_{c}$ or $T_{h}$ [2] and $k_{B}$ the Boltzmann's constant. At the adiabats $\mathcal{L}=\mathcal{L}_{N}$, which represents external noise in our controls. Any realistic refrigerator is subject to fluctuations in the external control which induces noise. The main point of this paper is to study the effects of noise on the behavior of the Otto cycle. First we consider the phase noise; it occurs when a piecewise process controls the scheduling of $\omega$ in time. For such a procedure random errors are expected in the duration of the time intervals described by the Linbland superoperator $\mathcal{L}_{N}$. We describe these errors by a Gaussian white noise which mathematically is equivalent to a dephasing process on the adiabats [10]. Then the dissipative operator $\mathcal{L}_{N}$ is given by $[9,11]$

$$
\mathcal{L}_{N_{p}}(\hat{\mathbf{A}})=-\frac{\gamma_{p}}{\hbar^{2}}[\hat{\mathbf{H}},[\hat{\mathbf{H}}, \hat{\mathbf{A}}]]
$$

In addition to the phase noise there is a more obvious source of external noise which is induced by fluctuations in the control frequency $\omega(t)$; this term represents Markovian random fluctuations in the profile of the harmonic oscillator. 
The dissipative Linbland term is

$$
\mathcal{L}_{N_{a}}(\hat{\mathbf{A}})=-\gamma_{a} \omega^{2}[\hat{\mathbf{B}},[\hat{\mathbf{B}}, \hat{\mathbf{A}}]]
$$

where $\hat{\mathbf{B}}=m \omega \hat{\mathbf{Q}}^{2} /(2 \hbar)$. A more detailed description of the four segments is as follows.

Hot isochore. The frequency is constant $\omega=\omega_{h}$ and the working medium is in contact with the hot bath at temperature $T_{h}$. In this case $\mathcal{L}=\mathcal{L}_{D}$ is the dissipative Lindblad term, Eq. (3), which leads the system toward thermal equilibrium of an harmonic oscillator defined by $k_{\uparrow} / k_{\downarrow}=\exp \left[-\hbar \omega /\left(k_{B} T\right)\right]$ [2]. The dynamics of the segment are described by the operator $\mathcal{U}_{h}$

Expansion adiabat. The working medium is isolated from the baths and the frequency $\omega(t)$ changes from the initial value $\omega_{0}=\omega_{h}$ to the final one $\omega_{f}=\omega_{c}$. The Liouville superoperator is $\mathcal{L}=\mathcal{L}_{N}$ which represents external noise in our controls. The propagator $\mathcal{U}_{h c}$ associated with this branch is our main subject of study.

Cold isochore. The frequency is maintained constant $\omega=$ $\omega_{c}$ and the working medium is in contact with the cold bath at temperature $T_{c} . \mathcal{L}$ is again a dissipative Lindblad term $\mathcal{L}_{D}$. The evolution operator is $\mathcal{U}_{c}$.

Compression adiabat. The frequency $\omega(t)$ changes from the initial value $\omega_{c}$ to the final one $\omega_{h}$. The Liouville superoperator $\mathcal{L}=\mathcal{L}_{N}$ represents the external noise. The dynamic is described by $\mathcal{U}_{c h}$. Then the total propagator associated with one whole cycle is the product

$$
\mathcal{U}_{\text {cyc }}=\mathcal{U}_{c h} \mathcal{U}_{c} \mathcal{U}_{h c} \mathcal{U}_{h}
$$

The state that describes the system is fully characterized by the thermodynamical variables. Statistical thermodynamics define that the state is determined by the maximum entropy condition subject to the constraints imposed by the thermodynamical observables [12-14]. At thermal equilibrium the energy expectation value is sufficient to represent the state of the system, maximizing the von Neumann entropy $S_{\mathrm{vN}}=-k_{B} \operatorname{Tr}[\hat{\rho} \ln (\hat{\rho})]$ subject to the energy constraint sets the density matrix at thermal equilibrium [14],

$$
\hat{\rho}_{\mathrm{eq}}=\frac{1}{Z} e^{-\hat{H} /\left(k_{B} T\right)}
$$

where $Z=\operatorname{Tr}\left[e^{-\hat{H} /\left(k_{B} T\right)}\right]$ is the partition function. However, during the cycle the system is not in general at thermal equilibrium, thus in order to generalize the canonical form Eq. (7) additional thermodynamical observables are necessary to characterize the state of the system. The density matrix of the state that maximizes the entropy subject to the constraints $\left\langle\hat{\mathbf{A}}_{i}\right\rangle=\operatorname{Tr}\left[\hat{\mathbf{A}}_{i} \hat{\rho}\right]$ is

$$
\hat{\rho}=\frac{1}{Z} e^{\sum_{i} \beta_{i} \hat{\mathbf{A}}_{i}}
$$

where $\beta_{i}$ are the Lagrange multipliers. This generalized canonical form is meaningful if the state can cast Eq. (8) during the whole cycle leading to canonical invariance $\beta_{j}=\beta_{j}(t)$ [14] which is guaranteed if the set of operators $\hat{\mathbf{A}}_{i}$ of Eq. (8) is closed under the dynamics generated by the equation of motion, Eq. (2). For the part $i[\hat{\mathbf{H}}, \hat{\mathbf{A}}] / \hbar+\partial \hat{\mathbf{A}} / \partial t$ which produces a unitary evolution it occurs that the Hamiltonian of the system is a linear combination of the operator set $\hat{\mathbf{A}}_{i}, \hat{\mathbf{H}}(t)=\sum_{m} h_{m} \hat{\mathbf{A}}_{m}$ (where $h_{m}$ are the expansion coefficients) and the set $\hat{\mathbf{A}}_{i}$ forms a closed Lie algebra $\left[\hat{\mathbf{A}}_{i}, \hat{\mathbf{A}}_{j}\right]=\sum_{k} C_{i j k} \hat{\mathbf{A}}_{k}$ (the $C_{i j k}$ are the structure factors) that preserves canonical invariance [15]. For the Otto cycle the set of thermodynamical observables $\hat{\mathbf{A}}^{\prime}=\left\{\hat{\mathbf{P}}^{2}, \hat{\mathbf{Q}}^{2}, \hat{\mathbf{Q}} \hat{\mathbf{P}}+\hat{\mathbf{P}} \hat{\mathbf{Q}}\right\}$ forms a SU(1,1) closed Lie algebra. In addition, for the nonunitary part $\mathcal{L}$ of Eq. (2) this set of three thermodynamical observables is closed to either $\mathcal{L}_{D}, \mathcal{L}_{N_{p}}$, or $\mathcal{L}_{N_{a}}$, which is sufficient to preserve canonical invariance [2]. The choice of this set of thermodynamical observables is not unique; in particular, we consider the following three $\hat{\mathbf{A}}=$ $\{\hat{\mathbf{H}}, \hat{\mathbf{L}}, \hat{\mathbf{D}}\}$ time dependent operators: $\hat{\mathbf{H}}=\frac{1}{2 m} \hat{\mathbf{P}}^{2}+\frac{1}{2} m \omega(t)^{2} \hat{\mathbf{Q}}^{2}$ Hamiltonian, $\hat{\mathbf{L}}=\frac{1}{2 m} \hat{\mathbf{P}}^{2}-\frac{1}{2} m \omega(t)^{2} \hat{\mathbf{Q}}^{2}$ Lagrangian, and $\hat{\mathbf{D}}=$ $\frac{1}{2} \omega(t)(\hat{\mathbf{Q}} \hat{\mathbf{P}}+\hat{\mathbf{P}} \hat{\mathbf{Q}})$ momentum-space correlation which satisfy the $\operatorname{SU}(1,1)$ algebra. The invariant Casimir operator associated with this algebra is $\hat{\mathbf{C}}=\left(\hat{\mathbf{H}}^{2}-\hat{\mathbf{L}}^{2}-\hat{\mathbf{D}}^{2}\right) /\left(\hbar^{2} \omega^{2}\right)$; recently it has been used to solve the dynamics of the system [16]. The closed algebra leads to closed equations of motion on the adiabats and on the isochores [3,17].

To make explicitly the connection between the state represented by the density matrix $\hat{\rho}$ and the expectation values of the three operators that guaranteed the canonical invariance let us rewrite the density matrix as [2]

$$
\hat{\rho}=\frac{1}{Z} e^{\alpha \hat{a}^{2}} e^{-\beta \hat{H}} e^{\alpha^{*} \hat{a}^{t^{2}}},
$$

where $*$ represents the complex conjugate. The Hermiticity of the density matrix $\hat{\rho}=\hat{\rho}^{\dagger}$ imposes the real and complex nature of the time dependent Lagrange multipliers $\beta \in \mathbb{R}$ and $\alpha \in \mathbb{C}$. In terms of the expectation values of the thermodynamical observables $\left\langle\hat{\mathbf{A}}_{i}\right\rangle$ the Lagrange multipliers are given by [2]

$$
\begin{gathered}
\alpha=\frac{2 \hbar \omega[\langle\hat{\mathbf{L}}\rangle+i\langle\hat{\mathbf{D}}\rangle]}{4\left[\langle\hat{\mathbf{L}}\rangle^{2}+\langle\hat{\mathbf{D}}\rangle^{2}\right]-[\hbar \omega-2\langle\hat{\mathbf{H}}\rangle]^{2}}, \\
\beta=\frac{1}{\hbar \omega} \ln \left[\frac{4\left[\langle\hat{\mathbf{D}}\rangle^{2}-\langle\hat{\mathbf{H}}\rangle^{2}+\langle\hat{\mathbf{L}}\rangle^{2}\right]+\hbar^{2} \omega^{2}}{4\left[\langle\hat{\mathbf{L}}\rangle^{2}+\langle\hat{\mathbf{D}}\rangle^{2}\right]-[\hbar \omega-2\langle\hat{\mathbf{H}}\rangle]^{2}}\right] .
\end{gathered}
$$

\section{EQUATIONS OF MOTION}

The dynamics generated on the isochores is obtained from Eq. (2):

$$
\frac{d}{d t} \underbrace{\left(\begin{array}{c}
\hat{\mathbf{H}} \\
\hat{\mathbf{L}} \\
\hat{\mathbf{D}}
\end{array}\right)}_{=: \hat{\mathbf{A}}(t)}=\left(\begin{array}{ccc}
-\Gamma & 0 & 0 \\
0 & -\Gamma & -2 \omega \\
0 & 2 \omega & -\Gamma
\end{array}\right) \hat{\mathbf{A}}(t)+\left(\begin{array}{c}
\Gamma\langle\hat{\mathbf{H}}\rangle_{e q} \\
0 \\
0
\end{array}\right),
$$

where $\Gamma=k_{\downarrow}-k_{\uparrow}$ is the heat conductance and $\langle\hat{\mathbf{H}}\rangle_{\mathrm{eq}}=$ $\hbar \omega \operatorname{coth}\left[\hbar \omega /\left(2 k_{B} T\right)\right] / 2$ is the equilibrium expectation energy. The equation of motion for the Hamiltonian is decoupled from the equations for $\hat{\mathbf{L}}$ and $\hat{\mathbf{D}}$ and displays an exponential approach to equilibrium. In contrast the Lagrangian and momentum-space correlation operators show an oscillatory decay to an expectation value of zero at equilibrium. More explicitly, as in the isochores the frequency is constant, and 
the above equations can be integrated,

$$
\begin{gathered}
\hat{\mathbf{H}}(t)=e^{-t \Gamma}\left[\hat{\mathbf{H}}(0)-\hat{\mathbb{I}}\left\langle\hat{\mathbf{H}}_{\mathrm{eq}}\right\rangle\right]+\hat{\mathbb{I}}\left\langle\hat{\mathbf{H}}_{\mathrm{eq}}\right\rangle, \\
\hat{\mathbf{L}}(t)=e^{-t \Gamma}[\cos (2 \omega t) \hat{\mathbf{L}}(0)-\sin (2 \omega t) \hat{\mathbf{D}}(0)], \\
\hat{\mathbf{D}}(t)=e^{-t \Gamma}[\sin (2 \omega t) \hat{\mathbf{L}}(0)+\cos (2 \omega t) \hat{\mathbf{D}}(0)],
\end{gathered}
$$

with $\hat{\mathbf{H}}(0), \hat{\mathbf{L}}(0)$, and $\hat{\mathbf{D}}(0)$ the initial values of the operators.

For the case of the adiabats the equations of motion take the form

$$
\frac{d \hat{\mathbf{A}}(t)}{\omega d t}=\left(\begin{array}{ccc}
\mu+\gamma_{a} \omega & -\mu-\gamma_{a} \omega & 0 \\
-\mu+\gamma_{a} \omega & \mu-\left(4 \gamma_{p}+\gamma_{a}\right) \omega & -2 \\
0 & 2 & \mu-4 \gamma_{p} \omega
\end{array}\right) \hat{\mathbf{A}}(t),
$$

where the three operators and $\omega$ depend on $t$ and $\mu=\dot{\omega} / \omega^{2}$ is defined as a dimensionless adiabatic parameter [3]. The solution of these coupled differential equations depends on the functional form of $\omega(t)$. Now our main purpose is to solve Eq. (16) for the adiabats. From this expression we write the previous $3 \times 3$ matrix $\mathcal{M}$ as $\mathcal{M}=\mathcal{M}_{0}+\mathcal{N}_{p}+\mathcal{N}_{a}$, where $\mathcal{M}_{0}$ takes into account the noiseless evolution on the adiabats [3] and $\mathcal{N}_{p}$ and $\mathcal{N}_{a}$ are the contributions of the phase and amplitude noise, respectively:

$$
\begin{aligned}
& \mathcal{M}_{0}(t)=\left(\begin{array}{ccc}
\mu & -\mu & 0 \\
-\mu & \mu & -2 \\
0 & 2 & \mu
\end{array}\right), \\
& \mathcal{N}_{p}(t)=-4 \gamma_{p} \omega\left(\begin{array}{ccc}
0 & 0 & 0 \\
0 & 1 & 0 \\
0 & 0 & 1
\end{array}\right), \\
& \mathcal{N}_{a}(t)=\gamma_{a} \omega\left(\begin{array}{ccc}
1 & -1 & 0 \\
1 & -1 & 0 \\
0 & 0 & 0
\end{array}\right) .
\end{aligned}
$$

The solution for the noiseless evolution has been obtained before [3]. Usually the time evolution with an arbitrary functional form of $\omega(t)$ will involve quantum friction [2] due to the resultant parasitic increase in the internal energy. The dissipation of this energy, in particular into the cold bath, can cause the refrigerator to stop cooling. But it is known that there are some special functional forms of $\omega(t)$ that allow a frictionless evolution in the adiabats [3,4,18-21]. In particular, we consider the $\mu=$ const [3] case, as we will see below it sets frictionless dynamics under particular conditions. The frequency profile is

$$
\omega(t)=\omega_{0} /\left(1-\mu \omega_{0} t\right),
$$

with $\omega_{0}$ the initial frequency at the adiabats. To get the noiseless solution for constant $\mu$ we seek a solution of the type $\mathcal{U}_{h c}^{0}=\mathcal{U}_{1} \mathcal{U}_{2}$ where $\left[\mathcal{U}_{1}, \mathcal{U}_{2}\right]=0$ and with $\mathcal{U}_{1}$ the part associated with the adiabatic evolution. The operator $\mathcal{U}_{1}$ is obtained by changing the time variable $d \theta=\omega(t) d t$ and factoring out the term $\mu \hat{\mathbb{I}}$ in $\mathcal{M}_{0}$, thus

$$
\mathcal{U}_{1}=\frac{\omega(t)}{\omega_{0}} \hat{\mathbb{I}}, \quad \omega_{0}=\omega(t=0) .
$$

The nonadiabatic contribution is codified into the $\mathcal{U}_{2}$ operator which is obtained by diagonalizing the extra-diagonal terms for $\mu<-2$ :

$$
\mathcal{U}_{2}=\frac{1}{\Omega^{2}}\left(\begin{array}{ccc}
4-c \mu^{2} & -\mu \Omega s & -2 \mu(c-1) \\
-\mu \Omega s & \Omega^{2} c & -2 \Omega s \\
2 \mu(c-1) & 2 \Omega s & 4 c-\mu^{2}
\end{array}\right),
$$

where $\Omega=\sqrt{4-\mu^{2}}, c=\cos (\Omega \theta), s=\sin (\Omega \theta)$, and $\theta(t)=$ $-\ln \left(\frac{\omega_{0}}{\omega(t)}\right) / \mu$. From Eq. (20) we see that the propagator $\mathcal{U}_{2}$ induces mixing of $\hat{\mathbf{H}}, \hat{\mathbf{L}}$, and $\hat{\mathbf{D}}$. To characterize the deviation from perfect factorization of the three operators we define an adiabaticity measure $\delta[7]$ as

$$
\delta=\left[\mathcal{U}_{1}^{-1} \mathcal{U}_{h c}\right](1,1)-1,
$$

in this context of noiseless dynamics and constant $\mu, \delta=$ $\mathcal{U}_{2}(1,1)-1$. The propagator $\mathcal{U}_{2}$ is to be expanded around $\mu=$ 0 which corresponds to the adiabatic limit. For $\mu<-2, \mathcal{U}_{2}$ corresponds to a rotation matrix so $\hat{\mathbf{H}}, \hat{\mathbf{L}}$, and $\hat{\mathbf{D}}$ describe a periodic motion. Each period is defined by

$$
X=\Omega \theta=2 n \pi, \quad n=0,1,2,3 \ldots,
$$

thus at the end of each one $\mathcal{U}_{2}$ restores the identity matrix and the perfect adiabatic following conditions $\delta=0$ are satisfied, leading to frictionless dynamic. This frictionless condition sets a quantization on the constant adiabatic parameter $\mu$,

$$
\mu=\frac{-2 \ln \left(\frac{\omega_{0}}{\omega_{f}}\right)}{\sqrt{4 n^{2} \pi^{2}+\ln ^{2}\left(\frac{\omega_{0}}{\omega_{f}}\right)}},
$$

where $\omega_{f}$ is the final frequency at the adiabats. The frictionless allocating times in the adiabats can be calculated from Eqs. (18) and (23):

$$
\tau=\frac{\left(\frac{\omega_{0}}{\omega_{f}}-1\right) \sqrt{4 n^{2} \pi^{2}+\ln ^{2}\left(\frac{\omega_{0}}{\omega_{f}}\right)}}{2 \omega_{0} \ln \left(\frac{\omega_{0}}{\omega_{f}}\right)} .
$$

\section{EFFECT OF NOISE}

The propagators for both phase and amplitude noise are derived. For a constant $\mu$ the exact solutions can be obtained by numerical integration of Eq. (16). To gain insight the exact solution is compared to approximations which provide more transparent physical interpretations. To this end we consider separately the phase and amplitude noise in the adiabatic frictionless $\mu \rightarrow 0$ limit.

\section{A. Phase noise}

Now we include the phase noise through the dissipative operator $\mathcal{L}_{N_{p}}(\hat{\mathbf{A}})=-\frac{\gamma_{p}}{\hbar^{2}}[\hat{\mathbf{H}},[\hat{\mathbf{H}}, \hat{\mathbf{A}}]]$ and we seek a solution of the product form $\mathcal{U}_{h c} \stackrel{\hbar^{2}}{=} \mathcal{U}_{1} \mathcal{U}_{2} \mathcal{U}_{3}$. The equations of motion for $\mathcal{U}_{3}$ are obtained from the interaction picture

$$
\begin{aligned}
\frac{d}{\omega d t} \mathcal{U}_{3 p}(t) & =\mathcal{U}_{2}(-t) \mathcal{N}_{p}(t) \mathcal{U}_{2}(t) \mathcal{U}_{3 p}(t) \\
& =\mathcal{W}_{p}(t) \mathcal{U}_{3 p}(t),
\end{aligned}
$$


where

$$
\mathcal{W}_{p}(t)=\frac{4 \gamma_{p} \omega(t)}{\Omega^{4}}\left(\begin{array}{ccc}
(c-1) \mu^{2}\left[\mu^{2}(1+c)-8\right] & \mu s\left(\mu^{2} c-4\right) \Omega & 2 \mu(c-1)\left(\mu^{2} c-4\right) \\
-\mu s\left(\mu^{2} c-4\right) \Omega & -\left[\mu^{2}\left(s^{2}-1\right)+4\right] \Omega^{2} & -2 \mu^{2} s(c-1) \Omega \\
-2 \mu(c-1)\left(\mu^{2} c-4\right) & -2 \mu^{2} s(c-1) \Omega & -\left[\mu^{2}\left(\mu^{2}-4 s^{2}-8 c\right)+16\right]
\end{array}\right) .
$$

The Magnus expansion [22] is employed to solve Eq. (25) which can be rewritten as $\frac{d \mathcal{U}_{3 p}(X)}{d X}=\mathcal{W}_{p}(X) \mathcal{U}_{3 p}(X) / \Omega$ and obtain the $n$ period propagator $\mathcal{U}_{3 p}(X=2 n \pi)$,

$$
\mathcal{U}_{3 p}(X=2 n \pi) \approx e^{B_{1}+B_{2}+\cdots},
$$

where $B_{1}=\int_{0}^{2 n \pi} d X \mathcal{W}_{a}(X) / \Omega, \quad B_{2}=\frac{1}{2} \int_{0}^{2 n \pi} \int_{0}^{X} d X d X^{\prime}$ $\left[\mathcal{W}_{a}(X), \mathcal{W}_{a}\left(X^{\prime}\right)\right] / \Omega^{2}$, and so on. The first order Magnus term leads to

$$
\begin{aligned}
B_{1 p}= & \frac{\gamma_{p} \omega_{h} \mu\left(-1+e^{2 n \pi \mu / \Omega}\right)}{-16+3 \mu^{2}} \\
& \times\left(\begin{array}{ccc}
-32 & -16 & -\frac{32}{\mu}-6 \mu \\
16 & -4+\frac{64}{\mu^{2}} & 6 \mu \\
\frac{32}{\mu}+6 \mu & 6 \mu & 12+\frac{64}{\mu^{2}}
\end{array}\right) .
\end{aligned}
$$

In the adiabatic limit we neglect the $O(\mu)$ terms in the nondiagonal and the $O(0)$ terms in the diagonal so

$$
\mathcal{U}_{3 p}(X=2 n \pi)=\left(\begin{array}{ccc}
e^{\gamma_{p} \mathcal{F} \mu} & 0 & 0 \\
0 & \frac{4}{4+\mu^{2}} e^{-4 \gamma_{p} \mathcal{F} / \mu} & 0 \\
0 & 0 & e^{-\gamma_{p} \mathcal{F} \Omega^{2} / \mu}
\end{array}\right),
$$

where $\mathcal{F}=-\frac{16 \omega_{h}}{\left(-16+3 \mu^{2}\right)}\left(-1+e^{2 n \pi \mu / \Omega}\right)$; then the frictionless parameter given by Eq. (21) for phase noise is
$\delta=U_{3}(1,1)-1$,

$$
\delta_{p} \approx-1+e^{\gamma_{p} \mathcal{F} \mu} .
$$

Notice that as $\mu \rightarrow 0$ the phase noise in the first order Magus approximation vanishes $\delta_{p} \rightarrow 0$.

When computing the second order Magnus expansion for phase noise we get for the adiabatic limit

$$
\mathcal{U}_{3 p}^{(2)}=\left(\begin{array}{ccc}
\cosh \beta & -\sinh \beta & 0 \\
-\sinh \beta & \cosh \beta & 0 \\
0 & 0 & 1
\end{array}\right)
$$

where $\beta=\frac{16 \omega_{h}^{2} \gamma_{p}^{2}}{\left(4+3 \mu^{2}\right)}\left(-1+e^{4 n \pi \mu / \Omega}\right)$, so $\delta_{p}^{(2)}=-1+\cosh \beta$. When $n \rightarrow \infty$ it sets a finite value for the influence of phase noise

$$
\delta_{p}^{(2)}=-1+\cosh \left[4 \gamma_{p}^{2}\left(\omega_{h}^{2}-\omega_{c}^{2}\right)\right] .
$$

\section{B. Amplitude noise}

We progress in a similar way to calculate the phase noise produced by the dissipative operator $\mathcal{L}_{N_{a}}(\hat{\mathbf{A}})=$ $-\gamma_{a} \omega^{2}[\hat{\mathbf{B}},[\hat{\mathbf{B}}, \hat{\mathbf{A}}]]$. In this case the interaction picture for $\mathcal{U}_{3 a}$ is

$$
\frac{d}{\omega d t} \mathcal{U}_{3 a}(t)=\mathcal{U}_{2}(-t) \mathcal{N}_{a}(t) \mathcal{U}_{2}(t) \mathcal{U}_{3 a}(t)
$$

with

$$
\mathcal{W}_{a}(t)=\frac{\gamma_{a} \omega(t)}{\Omega^{4}}\left(\begin{array}{ccc}
\left(4-\mu^{2} c+\mu s \Omega\right)^{2} & (\mu s+\Omega c)\left(-4+\mu^{2} c-\mu \Omega s\right) \Omega & 2(\mu-\mu c+\Omega s)\left(4-\mu^{2} c+\mu \Omega s\right) \\
(\mu s+\Omega c)\left(4-\mu^{2} c+\mu \Omega s\right) \Omega & -(\mu s+\Omega c)^{2} \Omega^{2} & 2(\mu s+\Omega c)(\mu-\mu c+\Omega s) \Omega \\
-2(\mu-\mu c+\Omega s)\left(4-\mu^{2} c+\mu \Omega s\right) & 2(\mu s+\Omega c)(\mu-\mu c+\Omega s) \Omega & -4(\mu-\mu c+\Omega s)^{2}
\end{array}\right) .
$$

In this case the first order Magnus term leads to

$$
\begin{aligned}
B_{1 a}= & \frac{\gamma_{a} \omega_{h}\left(-1+e^{2 n \pi \mu / \Omega}\right)}{-16+3 \mu^{2}} \\
& \times\left(\begin{array}{ccc}
-\frac{16}{\mu}+\mu & -\mu & 4 \\
\mu & \frac{8}{\mu}-\mu & 2 \\
4 & 2 & \frac{8}{\mu}
\end{array}\right),
\end{aligned}
$$

and in the adiabatic limit just the $O\left(\mu^{-1}\right)$ terms are considered, then

$$
\mathcal{U}_{3 a}(X=2 n \pi)=\left(\begin{array}{ccc}
e^{\gamma_{a} \mathcal{F} / \mu} & 0 & 0 \\
0 & e^{-\gamma_{a} \mathcal{F} /(2 \mu)} & 0 \\
0 & 0 & e^{-\gamma_{a} \mathcal{F} /(2 \mu)}
\end{array}\right),
$$

so the adiabaticity measure reads

$$
\delta_{a} \approx-1+e^{\gamma_{a} \mathcal{F} / \mu} .
$$

These two analytic expressions for $\delta$ match the exact values obtained numerically solving Eqs. (25) or (33) for phase and amplitude noise as we infer from Fig. 1. Amplitude and phase noise show the opposite behavior in the adiabatic limit of $n \rightarrow \infty$. Amplitude noise diverges when the process is more adiabatic, $\delta_{a} \sim 1 / \mu$, Eq. (37), whereas phase noise $\delta_{p}$ tends to a constant as $\mu \rightarrow 0$, Eq. (32).

\section{MINIMAL TEMPERATURE}

Does noise in the Otto cycle have any consequence on the performance of the refrigerator when $T_{c} \rightarrow 0$ ? Is there 


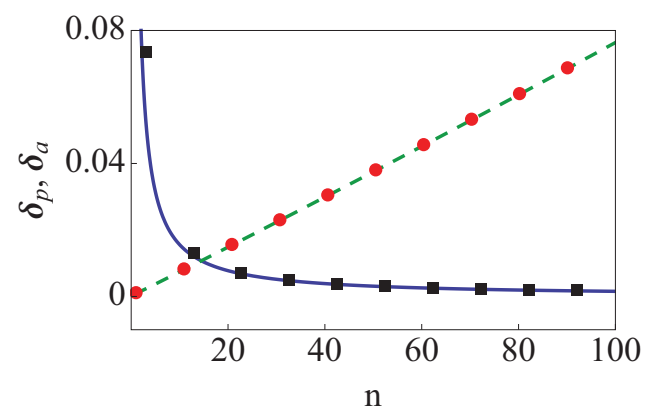

FIG. 1. (Color online) Parameter $\delta$ versus the frictionless cycle number $n$ for phase and amplitude noise. Blue solid line: approximated $\delta_{p}$ computed with the analytic Eq. (30). Black squares: exact $\delta_{p}$ solving numerically Eqs. (25) and (26). Green dashed line: approximated $\delta_{a}$ computed with the analytic Eq. (37). Red circles: exact $\delta_{a}$ solving numerically Eqs. (33) and (34). Parameter values: $\omega_{c}=2 \pi \times 1000 \mathrm{rad} / \mathrm{s}, \omega_{h} / \omega_{c}=25, \gamma_{p}=10^{-6} \mathrm{~s}^{-1}$, and $\gamma_{a}=5 \times 10^{-9} \mathrm{~s}^{-1}$.

a minimum temperature above the absolute zero where the refrigerator stops working?

The conditions for refrigeration require that on the cold side the internal energy of the working medium is smaller than the equilibrium energy with the cold bath at the end of the expansion adiabat,

$$
\langle\hat{\mathbf{H}}\rangle_{c} \leqslant\langle\hat{\mathbf{H}}\rangle_{\mathrm{eq}}\left(T_{c}\right)=\frac{\hbar \omega_{c}}{2} \operatorname{coth}\left(\frac{\hbar \omega_{c}}{2 k_{B} T_{c}}\right) .
$$

On the hot isochore the lowest temperature that can be obtained is in equilibrium

$$
\langle\hat{\mathbf{H}}\rangle_{h} \geqslant\langle\hat{\mathbf{H}}\rangle_{\mathrm{eq}}\left(T_{h}\right)=\frac{\hbar \omega_{h}}{2} \operatorname{coth}\left(\frac{\hbar \omega_{h}}{2 k_{B} T_{h}}\right) .
$$

Under these conditions of thermal equilibrium $L=D=0$ [2] and independently of what kind of noise we consider the change in $\langle\hat{\mathbf{H}}\rangle$ along the expansion adiabat is

$$
\frac{\langle\hat{\mathbf{H}}\rangle_{c}}{\omega_{c}} \approx(1+\delta) \frac{\langle\hat{\mathbf{H}}\rangle_{h}}{\omega_{h}}
$$

with $\delta$ the deviation from the perfect adiabatic following defined in Eq. (21). Then the maximum heat that can be extracted at the adiabats is given by

$$
\begin{aligned}
\mathcal{Q}(\max ) & =\langle\hat{\mathbf{H}}\rangle_{\mathrm{eq}}\left(T_{c}\right)-\langle\hat{\mathbf{H}}\rangle_{c} \\
& \approx \mathcal{Q}_{0}(\max )-\frac{\hbar \omega_{c}}{2} \delta \operatorname{coth}\left(\frac{\hbar \omega_{h}}{2 k_{B} T_{h}}\right),
\end{aligned}
$$

where $\mathcal{Q}_{0}(\max )=\frac{1}{2} \hbar \omega_{c}\left[\operatorname{coth}\left(\frac{\hbar \omega_{c}}{2 k_{B} T_{c}}\right)-\operatorname{coth}\left(\frac{\hbar \omega_{h}}{2 k_{B} T_{h}}\right)\right]$ is the maximum heat that can be extracted without noise [2]. Noise decreases the heat extraction efficiency. The condition for refrigeration is that $\mathcal{Q}(\max ) \geqslant 0$, then the temperature should satisfy

$$
T_{c} \geqslant \frac{\hbar \omega_{c}}{2 k_{B} \operatorname{arccoth}\left[(1+\delta) \operatorname{coth}\left(\frac{\hbar \omega_{h}}{2 k_{B} T_{h}}\right)\right]},
$$

where $\delta=\mathcal{U}_{3}(1,1)-1$. For a noiseless evolution $\delta=0$, it sets the Carnot limit

$$
T_{c} \geqslant \frac{\omega_{c}}{\omega_{h}} T_{h}
$$

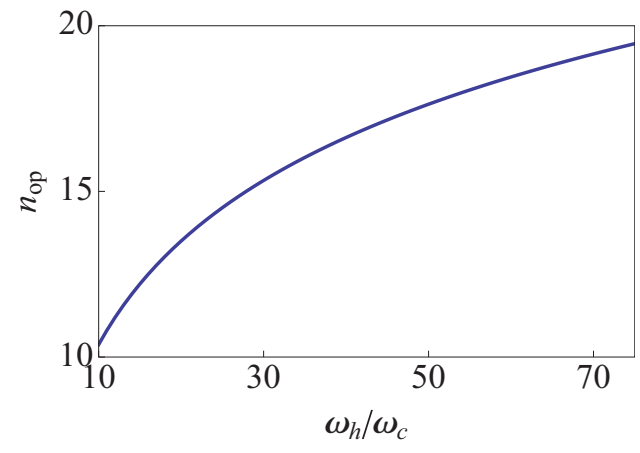

FIG. 2. (Color online) Optimum cycle $n_{\mathrm{op}}$ versus the ratio $\omega_{h} / \omega_{c}$. Same parameter values as in Fig. 1.

In contrast if phase or amplitude noise is present during the evolution the frictionless parameter $\delta$ is different from 0 , the value of $\delta$ in Eq. (42) should be replaced by Eq. (30) or (37) depending on what kind of noise is considered.

When both amplitude and phase noise are present there is an optimum cycle index $n$ which minimizes the temperature due to the two opposite tendencies when the process becomes more adiabatic (see Fig. 1). In that case the evolution operator $\mathcal{U}_{3}$ satisfies

$$
\begin{aligned}
\frac{d}{\omega d t} \mathcal{U}_{3 p a}(t) & =\mathcal{U}_{2}(-t)\left[\mathcal{N}_{p}(t)+\mathcal{N}_{a}(t)\right] \mathcal{U}_{2}(t) \mathcal{U}_{3 p a}(t) \\
& =\mathcal{W}_{p a}(t) \mathcal{U}_{3 p a}(t),
\end{aligned}
$$

where $\mathcal{W}_{p a}(t)=\mathcal{W}_{p}(t)+\mathcal{W}_{a}(t)$. Since the commutator $\left[U_{3 p}, U_{3 a}\right]=0$ then $U_{3 p a}=U_{3 p} U_{3 a}$ and as $U_{3 p}$ and $U_{3 a}$ are diagonals $U_{3 p a}(1,1)=U_{3 p}(1,1) U_{3 a}(1,1)$ or equivalently using the definition of Eq. $(21), U_{3 p a}(1,1)=\left(\delta_{p}+1\right)\left(\delta_{a}+1\right)$ so

$$
\delta_{p a} \approx \delta_{p}+\delta_{a}
$$

To find the optimum $n_{\text {op }}$ cycle we solve $\partial \delta_{p a} / \partial n=0$; the result is plotted in Fig. 2. This point $n_{\mathrm{op}}$ corresponds to the intersection $\delta_{p}\left(n_{\mathrm{op}}\right)=\delta_{a}\left(n_{\mathrm{op}}\right)$. Equating Eqs. (30) and (37) we find that $\mu\left(n_{\mathrm{op}}\right)=\sqrt{\gamma_{a} / \gamma_{p}}$ or explicitly using Eq. (23),

$$
n_{\mathrm{op}}=\frac{1}{2 \pi} \sqrt{\frac{4 \gamma_{p}}{\gamma_{a}}-1} \ln \left(\frac{\omega_{h}}{\omega_{c}}\right) .
$$

Replacing Eq. (46) in Eq. (30) or (37) we obtain the optimal $\delta$ value

$$
\delta_{p a}\left(n_{\mathrm{op}}\right)=-1+\exp \left(-\frac{16 \sqrt{\gamma_{a}} \gamma_{p}^{3 / 2}\left(\omega_{h}-\omega_{c}\right)}{3 \gamma_{a}-16 \gamma_{p}}\right) .
$$

Inserting this expression into Eq. (42) we get the minimum temperature

$$
T_{c} \geqslant \frac{\hbar \omega_{c}}{2 k_{B} \operatorname{arccoth}\left[\exp \left(-\frac{16 \sqrt{\gamma_{a}} \gamma_{p}^{3 / 2}\left(\omega_{h}-\omega_{c}\right)}{3 \gamma_{a}-16 \gamma_{p}}\right) \operatorname{coth}\left(\frac{\hbar \omega_{h}}{2 k_{B} T_{h}}\right)\right]} .
$$

We see that if $\omega_{c} \rightarrow 0$, then $T_{c} \rightarrow 0$, thus in contrast with reciprocating refrigerators which use different working mediums possessing a gap in their spectrum leading to a critical temperature in which they stop working due to the noise effects [5,6]. In this model by decreasing the gap $\omega_{c} \rightarrow 0$ 
the zero temperature $T_{c}=0$ is approachable even when the phase and amplitude noises are taken into account.

\section{CONCLUSIONS}

We have analyzed the influence of external noise on the behavior of the Otto cycle constituted by a quantum harmonic heat engine when it approaches the absolute zero. Phase and amplitude noise reduces the amount of heat that can be extracted per cycle showing a different behavior in the adiabatic limit, phase noise tends to a constant value whereas amplitude noise diverges. These two opposite tendencies set an optimal cycle for which the absolute zero can be approached, decreasing the energy gap $\omega_{c} \rightarrow 0$ when both kinds of noise are considered together.

The engine performance is examined for the particular trajectories $\mu=$ const which are frictionless when Eq. (22) is satisfied, however, these trajectories are not unique [18-21,23, $24]$ and in fact there are infinite possibilities for scheduling $\omega(t)$ which can be used to improve the quantum engine according to different criteria [25]. Given this freedom for choosing a frictionless trajectory, optimal control theory can be applied [3,4,26-28]. For noiseless evolution [3,4] bangbang trajectories where $\mu=$ const are the optimal to produce maximum work in the smallest time, how does noise modify these trajectories?

The dynamics of our reciprocating quantum refrigerator is codified into Eq. (17). Note that $\mathcal{M}_{0}(t)$ has an exceptional point $[29,30]$ at $\mu= \pm 2$ where all the eigenvalues and eigenvectors of the matrix collapse to the same one. A better physical understanding of these points and their relation with the speed limits are open questions.

\section{ACKNOWLEDGMENTS}

We are grateful to Y. Rezek and A. Levy for fruitful discussions. We acknowledge funding by Projects No. IT47210, No. FIS2009-12773-C02-01, No. FIS2012-36673-C0301, UPV/EHU program UFI 11/55, and the Israel Science Foundation (ISF).
[1] T. Jahnke, J. Birjukov, and G. Mahler, Ann. Phys. (Leipzig) 17, 88 (2008).

[2] Y. Rezek and R. Kosloff, New J. Phys. 8, 83 (2006).

[3] Y. Rezek, P. Salamon, K. H. Hoffman, and R. Kosloff, Europhys. Lett. 85, 30008 (2009).

[4] P. Salomon, K. H. Hoffman, Y. Rezek, and R. Kosloff, Phys. Chem. Chem. Phys. 11, 1027 (2009).

[5] T. Feldmann and R. Kosloff, Phys. Rev. E 70, 046110 (2004).

[6] T. Feldmann and R. Kosloff, Europhys. Lett. 89, 20004 (2010).

[7] R. Kosloff and T. Feldmann, Phys. Rev. E 82, 011134 (2010).

[8] G. Lindblad, Commun. Math. Phys. 48, 119 (1976).

[9] H. P. Breuer and F. Ptruccione, Open Quantum Systems (Oxford University Press, New York, 2002).

[10] T. Feldmann and R. Kosloff, Phys. Rev. E 73, 025107(R) (2006).

[11] V. Gorini and A. Kossakowski, J. Math. Phys. 17, 1298 (1976).

[12] E. T. Janes, Phys. Rev. 106, 620 (1957).

[13] E. T. Janes, Phys. Rev. 108, 171 (1957).

[14] A. Katz, Principles of Statistical Mechanics. The Information Theoretic Approach (Freeman, San Francisco, 1967).

[15] Y. Alhassid and R. D. Levine, Phys. Rev. A 18, 89 (1978).

[16] F. Boldt, J. D. Nulton, B. Andresen, P. Salamon, and K. H. Hoffmann, Phys. Rev. A 87, 022116 (2013).

[17] T. Feldmann and R. Kosloff, Phys. Rev. E 68, 016101 (2003).
[18] X. Chen, A. Ruschhaupt, S. Schmidt, A. del Campo, D. GuéryOdelin, and J. G. Muga, Phys. Rev. Lett. 104, 063002 (2010).

[19] E. Torrontegui, X. Chen, M. Modugno, A. Ruschhaupt, D. Guéry-Odelin, and J. G. Muga, Phys. Rev. A 85, 033605 (2012).

[20] E. Torrontegui, S. Ibáñez, S. Martínez-Garaot, M. Modugno, A. del Campo, D. Guéry-Odelin, A. Ruschhaupt, X. Chen, and J. G. Muga, Adv. At. Mol. Opt. Phys. 62, 117 (2013).

[21] A. del Campo, J. Goold, and M. Paternostro, arXiv:1305.3223.

[22] S. Blanes, F. Casas, J. A. Oteo, and J. Ros, Phys. Rep. 470, 151 (2009).

[23] E. Torrontegui, S. Ibáñez, Xi Chen, A. Ruschhaupt, D. GuéryOdelin, and J. G. Muga, Phys. Rev. A 83, 013415 (2011).

[24] C. Jarzynski, arXiv:1305.4967.

[25] J. Deng, Q. Wang, and J. Gong, arXiv:1305.4207.

[26] D. Stefanatos, J. Ruths, and J.-S. Li, Phys. Rev. A 82, 063422 (2010).

[27] E. Torrontegui, X. Chen, M. Modugno, S. Schmidt, A. Ruschhaupt, and J. G. Muga, New J. Phys. 14, 013031 (2012).

[28] X. Chen, E. Torrontegui, D. Stefanatos, J. S. Li, and J. G. Muga, Phys. Rev. A 84, 043415 (2011).

[29] T. Kato, Perturbation Theory of Linear Operators (Springer, Berlin, 1966).

[30] R. Uzdin, E. G. Dalla Torre, R. Kosloff, and N. Moiseyev, Phys. Rev. A 88, 022505 (2013). 